\title{
Restrictions on the injection energy of positrons annihilating near the Galactic center
}

\author{
D.O. Chernyshov*1,2,3, K.S. Cheng ${ }^{1}$, V.A. Dogiel ${ }^{2}$ \\ ${ }^{1}$ Department of Physics, University of Hong Kong, Pokfulam Road, Hong Kong, China \\ ${ }^{2}$ I.E.Tamm Theoretical Physics Division of P.N.Lebedev Institute, Leninskii pr, 53, 119991 \\ Moscow, Russia \\ ${ }^{3}$ Moscow Institute of Physics and Technology, Institutskii lane, 141700 Moscow Region, \\ Dolgoprudnii, Russia
}

\begin{abstract}
Beacom and Yüksel (2006) suggested an elegant method to estimate an initial energy of annihilating positrons from the ratio of $511 \mathrm{keV}$ line and in-flight annihilation fluxes. The idea was that in the case of stationary injection of positrons at high energies the fluxes of $511 \mathrm{keV}$ line emission (generated by thermalized positrons) and of in-flight annihilation (produced by fast positrons) were proportional to each other. For the delta-function injection spectrum they showed that the initial energy of annihilating positrons cannot exceed $3 \mathrm{MeV}$. Otherwise the expected in-flight annihilation flux of fast positrons in the range 1 to $10 \mathrm{MeV}$ is higher than observed by COMPTEL. However, the conclusion may be changed significantly if one analyzes the spatial distribution of the $511 \mathrm{keV}$ emission and 1-10 MeV emission. In addition if the magnetic field in Galactic center region is $1 \mathrm{mG}$ or higher the injection energy of positrons produced by central compact source can be unrestricted.
\end{abstract}

7th INTEGRAL Workshop

September 8-11 2008

Copenhagen, Denmark

\footnotetext{
* Speaker.
} 


\section{Introduction}

One of the interesting and still unsolved problems is the origin of $511 \mathrm{keV}$ annihilation emission from the Galactic Bulge. It is observed as an extended diffuse emission from $5^{\circ}-8^{\circ}$ radius region with the flux $\sim 0.7 \times 10^{-3} \mathrm{ph} \mathrm{cm}^{-2} \mathrm{~s}^{-1}$ that requires the rate of positron production there $\sim 10^{43} \mathrm{~s}^{-1}[1-3]$. These observations show that the energy of annihilating positrons is about several $\mathrm{eV}$. On the other hand, all potential sources of positrons in the Galaxy like SN stars [1], massive stars $\left({ }^{26} \mathrm{Al}\right.$-decay) [4], secondary electrons from p-p collisions [5], lepton jets of AGNs [6], dark matter annihilation[7], microquasars [8] etc. generate positrons with energies $\geq 1 \mathrm{MeV}$. This means that positrons should effectively lose their energy before annihilation and thus generate emission in other than $511 \mathrm{keV}$ energy ranges. Therefore, the injection energy of positrons is an essential parameter for modeling annihilation processes, and it can be in principle discriminated from observations.

Beacom and Yüksel [9] introduced a simple way to estimate the injection energy of positrons which is described below. We distinguish between different annihilation processes which are: inflight annihilation of high energy positrons which generates a continuum emission at energies above $511 \mathrm{keV}$ and annihilation of thermal positrons which are just responsible for the line emission. For the lifetime of in-flight annihilation, $\tau_{a n n}$, and average cooling time $\tau_{c l}$ of high energy positrons one can estimate the expected flux of in-flight annihilation, $I_{i f}$ from the observed $511 \mathrm{keV}$ line emission, $I_{\text {line }}$, as

$$
I_{i f}=\frac{\tau_{c l}}{\tau_{\text {ann }}} I_{\text {line }}
$$

where

$$
\tau_{a n n}=\left(n \int_{E_{i n j}}^{E_{\gamma}} \sigma_{a n n}(E) v(E) d E\right)^{-1} \text { and } \tau_{c l}=\int_{E_{i n j}}^{E t h} \frac{d E}{(d E / d t)_{c l}}
$$

Here $E_{\gamma}, E_{i n j}, E_{t h}$ and $v(E)$ are the energy of in-flight photons, the injection energy of positrons, the energy of thermal plasma, and the positron velocity, respectively. The plasma density is denoted as $n$ and $(d E / d t)_{c l}$ is the rate of energy losses defined as sum of ionization and synchrotron losses:

$$
(d E / d t)_{c l}=(d E / d t)_{i o n}+(d E / d t)_{s y n}
$$

Assuming that cooling of positrons is only due to ionization losses and taking into account that the both timescales are proportional to medium density one can notice that relation (1.1) is independent of the medium density. So this restrictions is universal and can be applied even to a medium with unknown density.

In the above-mentioned models the injection energy of positrons is expected in the range from 1 to several tens $\mathrm{MeV}$. Therefore, the in-flight gamma-ray emission is also expected in this energy range. The central part of the Galaxy in the range of several tens MeV was observed with COMPTEL. The origin of this emission is still unclear since known processes of gamma-ray production (like inverse Compton, bremsstrahlung etc.) are unable to generate the observed flux [10], [11].

Beacom and Yüksel [9] assumed that just this excess put a nice restriction on the positron injection energy. For any injection energy the in-flight gamma-ray flux has not to exceed the COMPTEL data. However, this excess above theoretical estimates is observed not only in the direction of the 
Galactic center. It is almost constant along the Galactic disk [12] where the intensity of annihilation emission is lower than in the Galactic center that makes the in-flight interpretation of the excess rather doubtful.

Therefore, Sizun et al. [7] suggested a more rigid constraint on the in-flight gamma-ray flux from the Galactic center. According to their criterion it should not exceed two statistical errors of COMPTEL measurements.

From the analysis of Beacom and Yüksel [9] and Sizun et al. [7] it follow that the injection energy of positrons should equal or be smaller than $1-3 \mathrm{MeV}$. Hence, all models which require higher injection energy of positrons, like e.g. that of Cheng et al. [5], should be rejected.

Below we show, nevertheless, that the injection energy may be much higher than $1-3 \mathrm{MeV}$. We analyze separately the criterion obtained by Beacom and Yüksel (2006) and Sizun et al. (2006).

\section{Medium properties in the vicinity of the Galactic center}

As follows from observations the central $200 \mathrm{pc}$ part of the Galaxy is strongly nonuniform. The inner bulge (200-300 pc) contains $(7-9) \times 10^{7} M_{\odot}$ of hydrogen gas. In spite of relatively small radius this region contains about $10 \%$ of the Galaxy's molecular mass. Most of the molecular gas is contained in very compact clouds of mass $10^{4}-10^{6} M_{\odot}$, average densities $\geq 10^{4} \mathrm{~cm}^{-3}$.

However, this molecular gas occupies a rather small part of the central region, most of which is filled with a very hot gas. ASCA [13] measured the X-ray spectrum in the inner $150 \mathrm{pc}$ region which exhibited a number of emission lines from highly ionized elements which are characteristics for a $8-10 \mathrm{keV}$ plasma with the density $0.4 \mathrm{~cm}^{-3}$. Later on Chandra observations [14] showed an intensive X-ray emission at the energy $E_{x} \sim 8 \mathrm{keV}$ from the inner $20 \mathrm{pc}$ of the Galaxy. The plasma density was estimated in limits $0.1-0.2 \mathrm{~cm}^{-3}$. Recent SUZAKU measurements $6.9 / 6.7 \mathrm{keV}$ iron line ratio [15] was naturally explained by a thermal emission of $6.5 \mathrm{keV}$-temperature plasma.

From polarization measurements it was inferred that near the Galactic center a large scale magnetic fields is observed inside clouds and in the intercloud medium. The magnetic field strength is estimated by the value of several $\mathrm{mG}$ (see e.g.[16]).

In this case synchrotron losses become essential for positrons even in the energy range 3-30 $\mathrm{MeV}$. One can see from Fig. 1 that if magnetic field strength as high as $\mathrm{B}=3 \mathrm{mG}$ the cooling time of $1 \mathrm{GeV}$ positrons is the same as of positrons with energies about $1 \mathrm{MeV}$ experiencing only ionization losses.

\section{Model description}

The spectrum of positrons $F(p)$ in the Galactic central region (integrated over the emission volume) as a function of particle dimensionless momentum $p=p / \sqrt{m_{e} k T}$ ( $m_{e}$ is the mass of the positron and $\mathrm{T}$ is medium temperature) can be calculated from the equation presented in [17]:

$$
\frac{1}{p^{2}} \frac{d}{d p}\left[\mathscr{A}(p) \frac{d F}{d p}+\mathscr{B}(p) F\right]+\frac{F}{\tau_{a n n}}=Q(p)
$$

where $\mathscr{A}(p)$ is the momentum diffusion coefficient which is responsible for formation of Maxwellian distribution, $\mathscr{B}(p)$ describes total energy losses, $\tau_{a n n}$ is the characteristic time of annihilation losses 


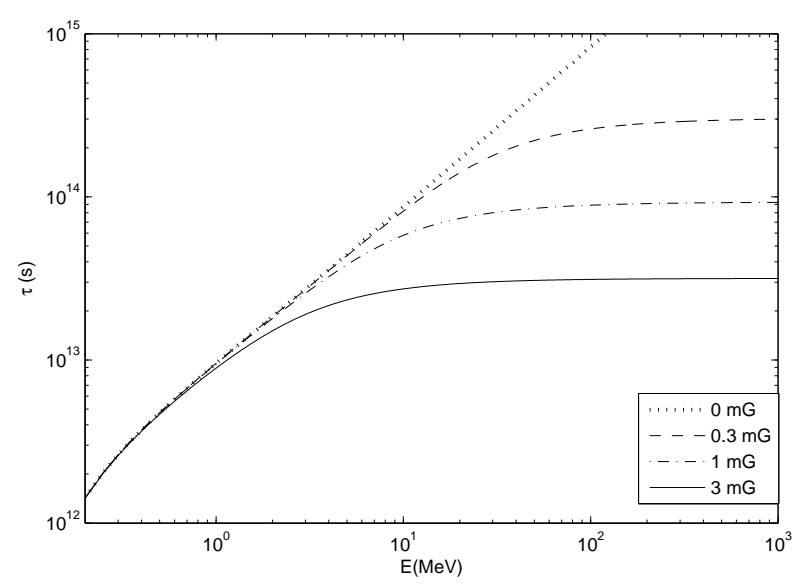

Figure 1: Characteristic cooling times of positrons for different values of magnetic field strength.

and $\mathrm{Q}(\mathrm{p})$ is the injection spectrum of the positrons. The exact values of $\mathscr{A}(p)$ and $\mathscr{B}(p)$ are presented in $[5,17]$.

The annihilation term consists of two parts:

$$
\frac{1}{\tau_{a n n}}=n_{e} v\left(\sigma_{c e}+\sigma_{d a}\right)
$$

where $\mathrm{v}$ is velocity of positrons, $\sigma_{c e}$ is the charge-exchange annihilation cross-section (Bussard et al (1979) [18]) and $\sigma_{d a}$ is the cross-section of the direct annihilation ( see [19]).

We will define injection function as the delta-function

$$
Q(p)=Q \delta\left(p-p_{\text {inj }}\right)
$$

where $p_{i n j}$ corresponds to injection energy and Q is determined by the the observed flux of 511 $\mathrm{keV}$ annihilation line from the bulge:

$$
\int n_{e} \sigma_{c e} v F(p) d p \simeq 10^{43} \mathrm{ph} \mathrm{s}^{-1},
$$

where $\mathrm{v}$ is particle velocity, $\mathrm{F}(\mathrm{p})$ is the solution of eq. (3.1). After this procedure we can easily estimate the spectrum of direct annihilation emission as described in Aharonian and Atoyan (2000) [20]:

$$
I_{d a}\left(E_{\gamma}\right)=\int_{p_{0}\left(E_{\gamma}\right)}^{\infty} n_{e} q_{d a}\left(p, E_{\gamma}\right) v F(p) d p
$$

where $q_{d a}$ is the production spectrum of in-flight annihilation from [20].

\section{Results}

\subsection{Beacom and Yüksel criterion}

We will start from the initial problem when magnetic field is weak and can be excluded from the analysis. Beacom and Yüksel used for their analysis the annihilation flux coming from a central $5^{\circ}$ - diameter region. They described the spatial distribution of annihilation emission by the 

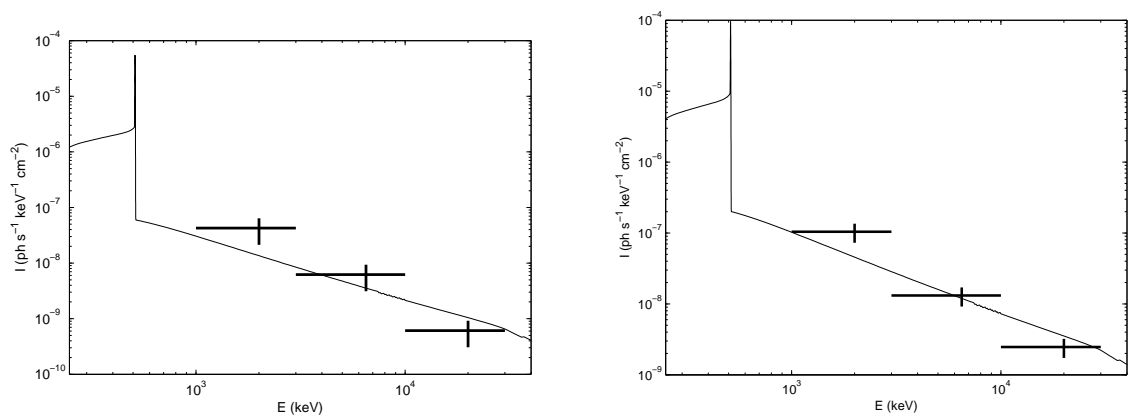

Figure 2: Annihilation spectrum for $|b|<2.5^{\circ},|l|<2.5^{\circ}$ (left) and $|b|<5^{\circ},|l|<5^{\circ}$ (right) together with COMPTEL data. $E_{\text {inj }}=30 \mathrm{MeV}, \mathrm{H}=0 \mathrm{mG}$

Gaussian with $\sigma=3.4^{\circ}$. For this distribution they derived that about $24 \%$ of the total annihilation flux comes from this region. Assuming that the COMPTEL flux in the energy range from 1 to 30 $\mathrm{MeV}$ taken from [10] was due to in-flight annihilation they concluded that the injection energy of annihilating positrons was equaled to or smaller than $1 \mathrm{MeV}$.

However, as follows from [1] the central $5^{\circ}$ - diameter region emits only $13 \%$ (not $24 \%$ ) of the total annihilation flux. We re-calculated the expected in-flight for $2.5^{\circ}$ - and $5^{\circ}$-radius radius regions for the injection energy of positrons is as high as $30 \mathrm{MeV}$. The $511 \mathrm{keV}$ annihilation flux was taken as observed. The results are shown in Fig. 2 together with the COMPTEL data taken from Strong et al [12].

One can see that within statistical uncertainties our analysis does not exclude the value of $30 \mathrm{MeV}$ as the injection energy of positrons. In this respect the secondary model of annihilating positrons seems to be probable. We notice, however, that the better coincidence between the COMPTEL data and the results of calculations were achieved for a broader area. The reason of this effect is evident from Fig. 3 where the longitudinal MeV distribution in the COMPTEL energy range is shown with the that of $511 \mathrm{keV}$ annihilation line. The last has a sharp peak in the direction of the galactic center. On the other hand statistical fluctuation of the COMPTEL flux are so large that they do not exclude a sharp peak of the $\mathrm{MeV}$ emission in this direction.

\subsection{Sizun et al. criterion}

Sizun et al. [7] made accurate calculations of the in-flight flux and showed that they did not contradict the COMPTEL data from [10] if the injection energy of positrons is about $10 \mathrm{MeV}$. They noticed, however, that the COMPTEL data did not show any significant excess in the bulge region in comparison with other part of the Galactic disk. Then to explain the origin of emission from the outer disk, one should add another (not in-flight) component of emission there which generate the same intensity of $\mathrm{MeV}$ emission there as that of positrons in the bulge. Such a hypothesis seems unrealistic.

The most plausible assumption would be that the COMPTEL emission is generated by other than in flight annihilation processes, while the in-flight flux from the bulge, if any, is small (within $2 \sigma$ of COMPTEL measurements). As Sizun et al. showed, with this restriction the upper bound 


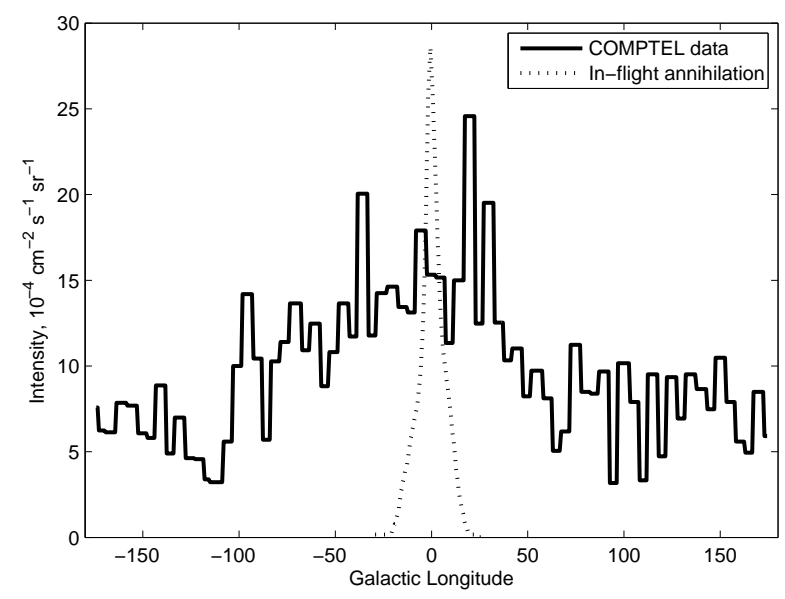

Figure 3: The distribution of the annihilation emission for $10 \mathrm{MeV}<\mathrm{E}<30 \mathrm{MeV}$ and $|b|<5^{\circ}$ together with COMPTEL data. $E_{i n j}=30 \mathrm{MeV}$

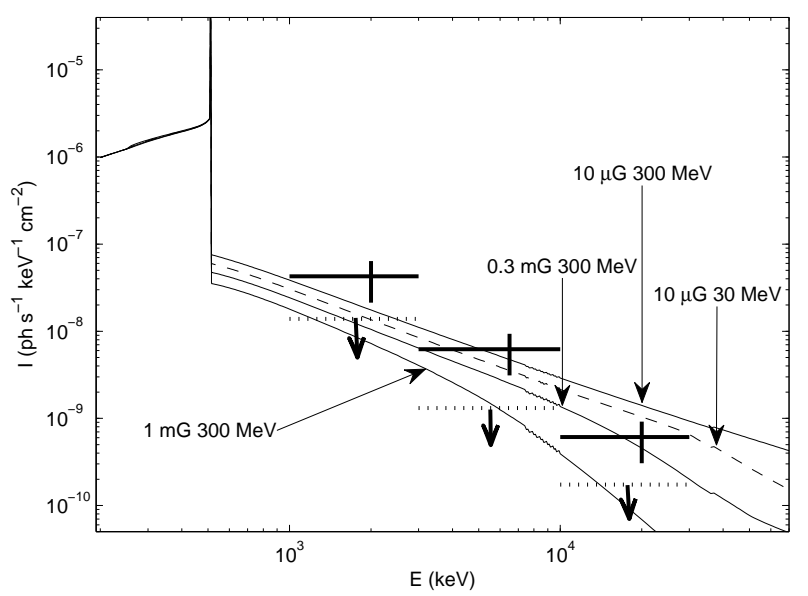

Figure 4: Annihilation spectrum for $|b|<2.5^{\circ}$ and $|l|<2.5^{\circ}$ and different values of injection energy and magnetic field. Solid crosses - spectrum measured by COMPTEL, dotted upper limits - statistical errors of measurement. Thin lines - spectrum of the annihilation emission.

of injection energy is between 3 and $7.5 \mathrm{MeV}$ that completely exclude the secondary origin of positrons.

We notice, however, that measurements show a very strong magnetic fields in the central region whose strength is as high as $3 \mathrm{mG}$. Then evolution of the positron spectrum is strongly influenced by synchrotron energy losses. With these magnetic fields ionization losses are essential when the initial energy of positrons drops to the value of $1 \mathrm{MeV}$ (see Fig. 1).

Calculations of in-flight flux from the bulge for different magnetic field strength together with the COMPTEL data for $2.5^{\circ} \times 2.5^{\circ}$ central area (solid crosses) and $2 \sigma$ statistical levels (dotted lines) are shown in Fig. 4. One can see that magnetic field strength as high as $1 \mathrm{mG}$ is enough to make calculations for any value of injection energy to be in a good agreement with observations. 


\section{Conclusion}

We have shown that under certain conditions such as relatively low medium density and strong magnetic field the injection energy of positrons in the Galactic center can be unrestricted. However, this model is valid only when a main part of positrons is born in regions with strong magnetic fields and stay there until they cool down to $1-3 \mathrm{MeV}$ and only then they leave this region. Because the region of strong magnetic field is relatively compact the fast positrons should be born in a close vicinity of the Galactic center that e.g. corresponds to the conditions of the model in [5]. It seems that this model can't allow heavy dark matter particles to be a source of positrons since the production region of positrons is extended. The next step of the analysis is to take into account spatial propagation of positrons. The solution of the equation with spatial terms will show the distribution of the annihilation emission along the Galactic axes so one can find whether the central source plays significant role in contribution to the annihilation emission or not.

\section{Acknowledgments}

We thank Andrew W. Strong for providing the COMPTEL data and for many useful discussions.

VAD and DOC are partly supported by the RFBR grant 08-02-00170-a, the NSC-RFBR Joint Research Project No 95WFA0700088 and by the grant of a President of the Russian Federation "Scientific School of Academician V.L.Ginzburg". KSC is supported by a RGC grant of Hong Kong Government under HKU 7014/07P.

\section{References}

[1] Knödlseder, J.; Jean, P.; Lonjou, V.; Weidenspointner, G.; Guessoum, N.; Gillard, W.; Skinner, G.; von Ballmoos, P.; Vedrenne, G.; Roques, J.-P.; Schanne, S.; Teegarden, B.; Schönfelder, V.; and Winkler, C., The all-sky distribution of 511 keV electron-positron annihilation emission, A\&A, 441, (2005), 513 [astro-ph/0506026]

[2] Churazov, E., Sunyaev, R., Sazonov, S., Revnivtsev, M., and Varshalovich, D., Positron annihilation spectrum from the Galactic Centre region observed by SPI/INTEGRAL, MNRAS, 1377, (2005), 1386 [astro-ph/0411351]

[3] Jean, P., Knödlseder, J., Gillard, W., Guessoum, N., Ferriere, K., Marcowith, A., Lonjou, V., and Roques, J. P., Spectral analysis of the Galactic e $+e$ - annihilation emission, A\&A, 445, (2006), 579 [astro-ph/0509298]

[4] Prantzos, N., On the intensity and spatial morphology of the $511 \mathrm{keV}$ emission in the Milky Way, $A \& A, 449,(2006), 869$ [astro-ph/0511190]

[5] Cheng, K. S., Chernyshov, D. O., \& Dogiel, V. A., Diffuse gamma-ray emission from the Galactic center - a multiple energy injection model, $A \& A, 473$, (2007), 351 [astro-ph/ 0706 . 4351]

[6] Totani T. A RIAF Interpretation for the Past Higher Activity of the Galactic Center Black Hole and the 511 keV Annihilation Emission, PASJ, 58, (2006), 965 [astro-ph/0607414]

[7] P. Sizun, M. Cassé and S. Schanne, Continuum $\gamma$-ray emission from light dark matter positrons and electrons, PhysRevD 74, 063514 (2006) [astro-ph/ 0607374 ]. 
[8] Weidenspointner, G., Skinner, G., Jean, P., Knödlseder, J., von Ballmoos, P., Bignami, G., Diehl, R., Strong, A. W., Cordier, B., Schanne, S., Winkler, Ch., An asymmetric distribution of positrons in the Galactic disk revealed by $\gamma$-rays, Nature, 451 (2008) 159

[9] J.F. Beacom and H. Yüksel, Stringent constraint on Galactic positron production, PhysRev Lett 97 , 071102 (2006) [astro-ph/0512411].

[10] Strong, A. W., Diehl, R., Halloin, H., Schönfelder, V., Bouchet, L., Mandrou, P., Lebrun, F., \& Terrier, R. Gamma-ray continuum emission from the inner Galactic region as observed with INTEGRAL/SPI, A\&A 444, (2005), 495

[11] Porter, T. A., Moskalenko I. V., Strong A.W., Orlando, E., Bouchet L., Inverse Compton Origin of the Hard X-Ray and Soft Gamma-Ray Emission from the Galactic Ridge, ApJ, 682, (2008), 400

[12] A.W. Strong, H. Bloemen, R. Diehl, W. Hermsen, V. Schönfelder, COMPTEL skymapping: a new approach using parallel computing, in proceeding of 3rd INTEGRAL Workshop (1998) [astro-ph/9811211v1]

[13] Koyama, K., Maeda, Y., Sonobe, T., Takeshima, T., Tanaka, Y., \& Yamauchi, S., ASCA View of Our Galactic Center: Remains of Past Activities in X-Rays?, PASJ 48, (1996), 249

[14] Muno, M. P., Arabadjis, J. S., Baganoff, F. K., Bautz, M. W., Brandt, W. N., Broos, P. S., Feigelson, E. D., Garmire, G. P., Morris, M. R., \& Ricker, G. R., The Spectra and Variability of X-Ray Sources in a Deep Chandra Observation of the Galactic Center, ApJ 613, (2004), 1179

[15] Koyama, K., Hyodo, Y., Inui, T., Nakajima, H., Matsumoto, H., Tsuru, T. G., Takahashi, T., Maeda, Y., Yamazaki, N. Y., Murakami, H., Yamauchi, S., Tsuboi, Y., Senda, A., Kataoka, J., Takahashi, H., Holt, S. S., \& Brown, G. V., Iron and Nickel Line Diagnostics for the Galactic Center Diffuse Emission, PASJ 59, (2007), 245

[16] M. Morris, The Galactic center magnetosphere, Journal of Physics: Conference Series 54, 1, (2006), 1 [astro-ph/0701050]

[17] Liang, H., Dogiel, V. A., \& Birkinshaw, M., The origin of radio haloes and non-thermal emission in clusters of galaxies, MNRAS 337, (2002), 567

[18] R.W. Bussard, R. Ramaty and R.J. Drachman, The annihilation of Galactic positrons, ApJ 228, (1979), 928

[19] C.J. Crannell, G. Joyce, R. Ramaty and C. Werntz, Formation of the 0.511 MeV line in solar flares, ApJ, 210, (1976), 210

[20] F.A. Aharonian, A.M. Atoyan, Broad-band diffuse gamma ray emission of the galactic disk, A\&A, 362, (2000), 937 [astro-ph/ 0009009 ]. 